\title{
Procedure and Installation for Determining the Filtering Capacity
}

\author{
Sorin Butuc (Anghel) ${ }^{1,}$, , Simona Matei $^{1}$, Maria Stoicanescu ${ }^{1}$, and Aurel Crişan ${ }^{1}$ \\ ${ }^{1}$ Transilvania University of Brasov, Material Science Department, 29 Eroilor Blvd, 500036, Brasov, \\ Romania
}

\begin{abstract}
The paper presents a method and an installation for determining the filtration capacity of filters made of composite materials with ceramic matrices. Applicable to fluid media (liquid, gaseous) the process is based on generating a pressure gradient in the filter area. The pressure difference between the two sides of the filter determines the passage of the fluid through the filter and its retention of the suspended particles. The process allows the testing of filters with a great diversity regarding the nature of the materials from which they are made, respectively their geometry and dimensions. In the present experiment, filters made of ceramic composite materials based on bentonite reinforced with $\mathrm{SiC}$ type ceramic powders and Al type metal powders were tested. From these materials were made cylindrical filters (diameters of $16 \mathrm{~mm}$ and length of $15 \mathrm{~mm}$ ), which, after sintering at a temperature of $1250^{\circ} \mathrm{C}$, were tested on the installation, for filtering rainwater samples. The determinations made highlighted a good ability of the filter to retain suspended particles in the water.
\end{abstract}

\section{Introduction}

Continuous industrialization and technological development have led to serious air, soil and water pollution in many regions of the world [1]. Increasingly, there is a growing interest in ensuring a healthy, viable, unpolluted environment. Chemicals $[2,3]$ or methods based on filtration processes $[3,4]$ can be used to purify water. In order to clarify the water, there is a tendency to use ecological process and methods. Natural water, due to the impurities contained in the form of particles of mineral or organic nature can be considered as a dispersed system of low concentration.

The substantial increase in the percentage of non-compliant waters is considered a major environmental problem [5]. Thus, a series of technologies have been studied and installations have been designed to filter water [6]. Laboratory -scale studies have been undertaken to investigate the applicability of a ceramic filter when introduced into a heterogeneous system $[3,6]$. It was provided efficient filtering performance [7]. Filtration of a heterogeneous liquid-solid medium can be done through ceramic filter [8-11], based on bentonite [13],

\footnotetext{
*Corresponding author: sorin.butuc@unitbv.ro
} 
cordierite [3] or even $\mathrm{CaO}-\mathrm{MgO}-\mathrm{SiO}_{2}$ fibres [12] which was interposed in a filtration plant, and the media were separated until the filter pores were loaded. Filtration plants can use ceramic filters to filter water without sacrificing their microbial removal effectiveness [14]. These filtration plants can operate on the basis of pressure gradient $[7,15,16]$ or with gascontrolled temperature furnace [14] or gravitational filtration takes place [3,17]. The installations facilitated the obtaining of a filtrate with improved physical-chemical properties. These filtering technologies and equipment [3, 7, 14-17], portable filtering devices [18], may have high installation and maintenance costs. This paper presents a method and an installation for determining the filtering capacity of filters made of composite materials with ceramic matrices.

The working principle involves the application of a pressure gradient on both sides of the filter that determines the passage of the fluid through the filter and its retention of suspended particles.

Determinations were made for certain materials and results were obtained. The purpose of this paper is to design a method and the necessary laboratory equipment to accurately measure the filtering capacity of ceramic matrix composites.

\section{Presentation of the installation}

In order to determine the filtration capacity, a filtration installation was designed and built (figure 1). The installation has in its component two parallelepiped glass tanks $(380 \mathrm{~mm}$ (Length) x $250 \mathrm{~mm}$ (Width) x $440 \mathrm{~mm}$ (Height)). Each tank has a useful volume of 401 . The tanks have been fixed neatly on a metal support that ensures strength and rigidity.

The installation is provided with a $\mathrm{Cu}$ pipe system with a diameter of $15 \mathrm{~mm}$. On the pipeline route of the installation are provided valve with $1 / 4$ inch ball that offers the possibility of controlled of the fluid, manometers for pressure control, flow meter for determining the flow of fluid, etc.

a)

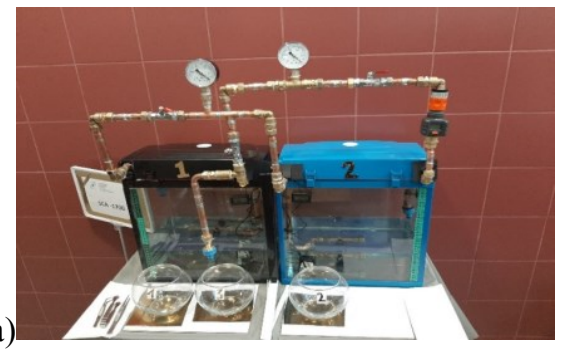

b)

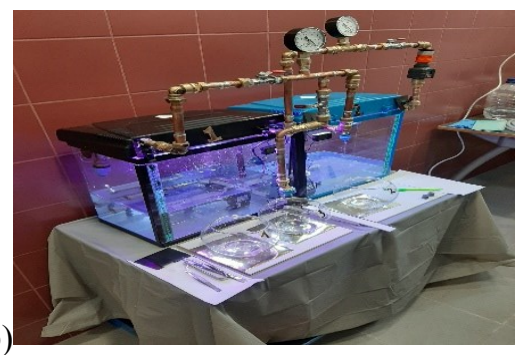

Fig. 1. Filter installation front view a) and side view b).

Each tank is equiped with centrifugal pump, and the characteristics that determine the performance of the installation are : maximum flow $\mathrm{Q}=15 \mathrm{l} / \mathrm{min}$, pump power $\mathrm{P}=7 \mathrm{~W}$, load height (pumping) $h_{\max }=0,95 \mathrm{~m}$, supply voltage $\mathrm{U}=230 \mathrm{~V}$, frequency $\mathrm{v}=50 \mathrm{~Hz}$, maximum temperature of the liquid conveyed by pump $\mathrm{t}=35^{\circ} \mathrm{C}$. The laboratory installation was bulit in order to render a filtartion process by means of a filter $(\phi=16 \mathrm{~mm}$ and $\mathrm{h}=15 \mathrm{~mm})$ made of composite materials. The centrifugal pump intervenes in the filtration process by generating a pressure gradient applied on both sides of the filter which causes the fluid to move. To ensure the stability of the filter during the filtration process, a filter holder was built. It was made of bronze by turning and drilling operations (figure 2).

The support was fixed on the route of the filtration installation in the suction area of the pump. 


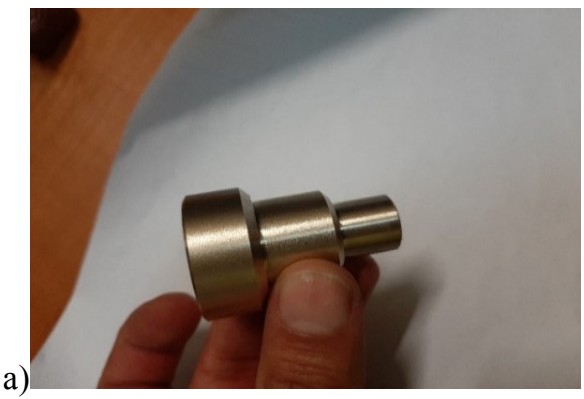

b)

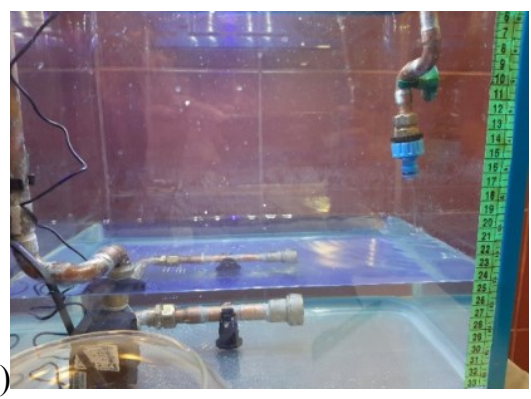

Fig. 2. Fixing support for ceramic filter a) and mounting the support in the installation b).

In order for the filtration process to take place in good conditions, the installation was provided with equipment that provides information about various parameters: digital flowmeter to determine the flow of fluid, pressure gauges to indicate pressure, lighting system, graduated scale for measuring the amount of fluid, etc. The functional diagram of the filtration installation is presented in figure 3 .

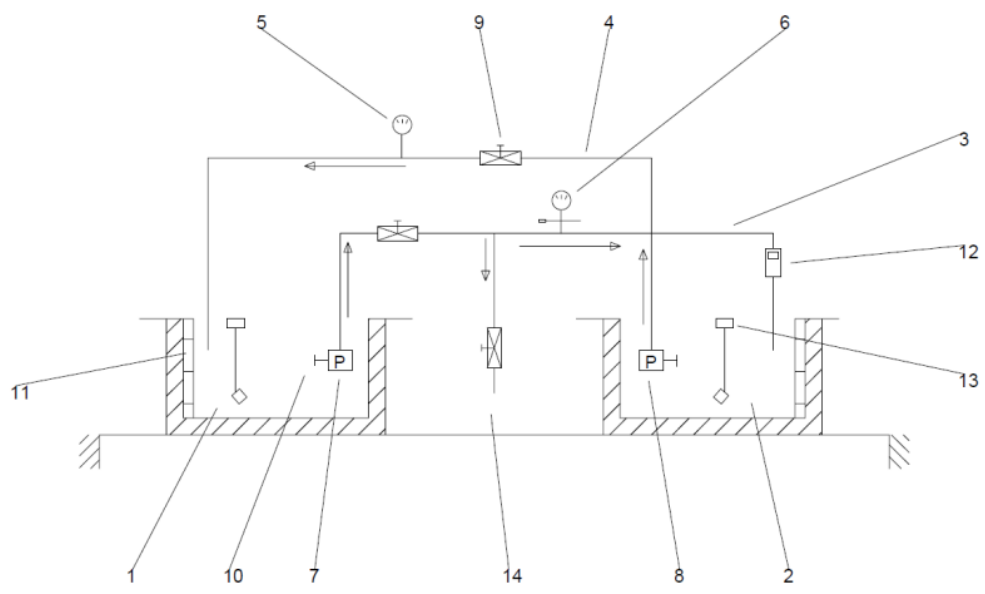

Fig. 3. Functional diagram of the filtration installation: 1,2- tank, 3- pipe route for pump no.1, 4- pipe route for pump no.2, 5,6- manometer, 7,8-centrifugal pump, 9- valve type operating valve with ball $1 / 4,10$ - filter mounting bracket, 11- graduated scale (in cm), 12- flow meter, 13-thermometer, 14- pipe route for fluid discharge.

The fluid in the tank 1 is forced to pass through ceramic filter fixed in the support 10 by means of centrifugal pump 7 and is transported to the tank 2 . The centrifugal pump 7 realizes a pressure gradient on both surfaces of ceramic filter which causes the fluid to move. The centrifugal pump converts mechanical energy into hydraulic energy. The fluid enters the slots of the rotor where it is accelerated, imprinting a rotational and a centrifugal movement [19]. It is thus pushed back with maximum speed. The energy characteristics of the pump are in turn influenced by the quality of the liquid conveyed, its characteristics (viscosity, concentration, specific gravity, working temperature) and the spatial configuration of the components installation (tank, suction line, location of the pump) [19]. The flow rate that the pump must convey in the installation, over a well- defined sector of it, can be measured by means of the digital flowmeter 12. For this, a predetermined volume of fluid is reported which 
is discharged over a period of time. The measurement of this volume can be performed using the graduated scale 11 located in the installation. Time is measured with a timer measuring and control device. The fluid has a temperature that can be indicated by means of digital thermometers with thermocouple 10, indicated in the diagram at position number 13 .

The use of measuring equipment, as well as those of distribution and control, offers the possibility of a process control. The process is hydrodynamic, and the phenomena that occur during filtration are influenced by factors physical, chemical, nature of the liquid circulated, type of filter used, load characteristics of the pump, etc. The pump circulates the liquid under a working pressure $p$, indicated by means of pressure gauges of the manometer type, mounted on the discharge pipes. They are sensitive and offer the possibility of indicating values in the range $0-1$ bar provided that a pressure environment is achieved (pressure discharge in a closed environment). The installation can be used in the following ways: stationary mode and mode with variable parameters. In steady state, the level of the liquid for filtration, respectively filtered, remains constant in the two tanks 1 and 2, with the help of level regulators. Tank 1 is constantly supplied with a certain flow of liquid for filtration, while from tank 2 an equal flow of filtered liquid is discharged. The value of this flow depends on the pump performance (maximum $15 \mathrm{l} / \mathrm{min}$ ). In the regime with variable parameters, a certain amount of liquid is transferred from tank 1 to tank 2 . Under these conditions, the flow of liquid passed through the filter varies during the process, depending on the level of liquid in the two tanks. The filtration process can continue for the same liquid by bringing it back from tank 2 to tank 1 . In both variants of using the installation, the filtration flow changes depending on the decrease of the filter permeability due to the retention of impurities in the liquid. The flow that the installation develops without the filter sample was called free flow. Relationship (1) returns free flow:

$$
V / t=\mathrm{Q}_{1}
$$

where $\mathrm{Q}_{1}$ was considered free flow and $\mathrm{t}$ represents the unit of time in which the volume $\mathrm{V}$ of fluid is discharged through the installation. The insertion of a filter in the installation support, determines the flow changes. The flow values vary depending on the time in which the pre-set fluid volume is discharged through the filtration systems. Relationship (2) shows a filtering start flow:

$$
V / t 1=\mathrm{Q}_{1}
$$

where $t_{1}$ represents the time in which the volume $\mathrm{V}$ of fluid is discharged through the sample, and $\mathrm{Q}_{1}$ represents the fluid flow at the beginning of filtration. After the complete discharge of the pre-set fluid volume, the filtration process continued and a new $\mathrm{Q}_{2}$ value of the flow rate given by relation (3) was registered.

$$
V / t 1+t x=\mathrm{Q}_{2}
$$

where $\mathrm{t} 1+\mathrm{t}_{\mathrm{x}}$ represents the time in which a volume $\mathrm{V}$ of fluid was further discharged. The filtering process continued through the filter and values of the times and implicitly of the new rates obtained were continuously recorded, until the flow rate decreased close to zero, obtaining an end of filtration flow rate.

\section{Determining the filtration efficiency}

Samples were made of ceramic composite materials based on bentonite. Bentonites have a high content of montmorillonite [20]. Bentonite has very good adsorption properties [13, 20]. 
Compacted [6], it has low permeability and is long-term stable. In the realization of the composite material, bentonite was used for the matrix and for reinforcement various ceramic powders of $\mathrm{SiC}$ (granulation $60-80 \mu \mathrm{m}$ ) and $\mathrm{Al}_{2} \mathrm{O}_{3}$ (granulation $50 \mu \mathrm{m}$ ) and metallic powders of $\mathrm{Al}$ (granulation 30-50 $\mu \mathrm{m}$ ) were used. Bentonite is from Suncuius area, the north-western part of Romania $\left(53 \%\right.$ montmorillonite $(\mathrm{Na}, \mathrm{Ca})_{0.33}(\mathrm{Al}, \mathrm{Mg})_{2}\left(\mathrm{Si}_{4} \mathrm{O}_{10}\right)(\mathrm{OH})_{2} \cdot \mathrm{nH}_{2} \mathrm{O} ; 1,3 \%$ $\mathrm{Fe}_{2} \mathrm{O}_{3} ; 45,7 \% \mathrm{SiO}_{2}$ ).

Bentonite was first mixed with $\mathrm{SiC}$ and $\mathrm{Al}_{2} \mathrm{O}_{3}$ in proportions of $40 / 60$ percent, thus obtaining two groups of materials. These were mixed for homogenization with the addition of $12 \%$ water in total for 20 minutes. To each of these two groups of compositions were then added amounts of $\mathrm{Al}$ powders ranging from 5-17\% mixing until complete homogenization. Table one shows the proportions of the components used for the tests.

Table 1. Structural composition of samples.

\begin{tabular}{|c|c|c|c|c|}
\hline Sample & Bentonite (\%) & $\mathrm{SiC}(\%)$ & $\mathrm{Al}_{2} \mathrm{O}_{3}(\%)$ & $\mathrm{Al}(\%)$ \\
\hline Sample 1 & 38 & 57 & - & 5 \\
\hline Sample 2 & 36 & 55 & - & 9 \\
\hline Sample 3 & 35 & 52 & - & 13 \\
\hline Sample 4 & 33 & 50 & - & 17 \\
\hline Sample 5 & 38 & - & 57 & 5 \\
\hline Sample 6 & 36 & - & 55 & 9 \\
\hline Sample 7 & 35 & - & 52 & 13 \\
\hline Sample 8 & 33 & - & 50 & 17 \\
\hline
\end{tabular}

The obtained mixtures were cold pressed in a metal mould at a pressure of $20 \mathrm{kN}$ for compaction in cylindrical samples and then sintered at a temperature of $1250{ }^{\circ} \mathrm{C}$ for 60 minutes in the oven atmosphere. The dimensions of these samples are $\phi=16 \mathrm{~mm} \mathrm{si} \mathrm{h}=15 \mathrm{~mm}$. Sintered, ceramic samples were subjected to stress [13]. On the universal mechanical testing machine, model WDW-150S the compressive strengths for the realized composites were determined. The universal testing machine allows determinations and tests. This machine allows the determination of mechanical properties such as tensile strength, compressive strength and bending strength. The test force can be between 1 and $150 \mathrm{kN}$. Following the requests, the following results were recorded, which are shown in table 2.

Table 2. Tests of compressive strength.

\begin{tabular}{|c|c|}
\hline Sample & Compressive strength (MPa) \\
\hline Sample 1 & 60 \\
\hline Sample 2 & 64 \\
\hline Sample 3 & 94 \\
\hline Sample 4 & 108 \\
\hline Sample 5 & 106 \\
\hline Sample 6 & 114 \\
\hline Sample 7 & 129 \\
\hline Sample 8 & 149 \\
\hline
\end{tabular}

In has been observed that increasing the percentage of $\mathrm{Al}$ metal particles in the bentonite and $\mathrm{Al}_{2} \mathrm{O}_{3}$ composite has led to a substantial improvement in mechanical properties by increasing the compressive strength.

The addition of ceramic particles of $\mathrm{SiC}$ and $\mathrm{Al}_{2} \mathrm{O}_{3}$ ensures the achievement of porous structure and by the additional addition of metallic particles of $\mathrm{Al}$ ensures a control of the pore size.

To determine the porosity, a method was used which consisted in measuring the volume of open pores. The sample was initially weighed using an analytical balance and the value of 
the mass $\mathrm{m}_{1}$ was recorded. Distilled water was introduced into a graduated cylinder and an initial volume $\mathrm{V}_{0}$ was measured. The sample was immersed in distilled water and volume $\mathrm{V}_{1}$ was measured after its introduction. For 30 minutes, the sample was kept in distilled water and the $V_{2}$ value of the volume of water in the graduated cylinder was recorded. Then, the sample was extracted from the water and weighed again by means of the analytical balance and the value $\mathrm{m}_{2}$ was recorded. The porosity of the sample, denoted by P1 was determined using the relation (4):

$$
\mathrm{P} 1=\frac{V 1-V 2}{V 1-V 0} 100
$$

The volume of water in the pores is indicated by the relation (5):

$$
\mathrm{V}_{\text {water in the pores }}=\frac{m 2-m 1}{\rho}
$$

The porosity of the sample was verified with the relation (6):

$$
\mathrm{P} 2=\frac{m 2-m 1 / \rho}{V 1-V 0}=\frac{m 2-m 1}{\rho(V 1-V 0)}
$$

Where $\rho=1 \mathrm{~g} / \mathrm{cm}^{3}$ was considered water density. Sample porosity values are shown in table 3.

Table 3. Sample porosity.

\begin{tabular}{|c|c|}
\hline Sample & Porosity (\%) \\
\hline Sample 1 & 14,14 \\
\hline Sample 2 & 17,44 \\
\hline Sample 3 & 16,20 \\
\hline Sample 4 & 13,95 \\
\hline Sample 5 & 13,57 \\
\hline Sample 6 & 14,44 \\
\hline Sample 7 & 18,60 \\
\hline Sample 8 & 9,82 \\
\hline
\end{tabular}

In experiment sample 2 was selected consisting of a ceramic composite material based on bentonite $(36 \%)$ reinforced with $\mathrm{SiC}$ ceramic particles $(55 \%)$ and $\mathrm{Al}$ particles $(9 \%)$. In order to choose the composition of the samples, several samples of mixtures were tried, the final variant being the one shown. The high degree of porosity determined the choice of this sample in the experiment. To determine the filtration efficiency of sample, its weight variation was taken into account. Before introducing the sample into the filtration process, it was dried in heat treatment furnace to remove the percentage of moisture. Heating was performed in the temperature range $40^{\circ} \mathrm{C}-100^{\circ} \mathrm{C}$ in 6 minutes, kept at $100^{\circ} \mathrm{C}$ for 15 minutes, followed by cooling from $100^{\circ} \mathrm{C}$ at $40^{\circ} \mathrm{C}$ for 6 minutes. The sample was weighed analytically and value $\mathrm{m}=7.05809 \mathrm{~g}$ was recorded. A predetermined volume of fluid $\mathrm{V}=1$ liter was discharged through the installation in a time of $\mathrm{t}=19,12 \mathrm{sec}$ resulting in the flow rate $\mathrm{Q}=$ $3,1381 / \mathrm{min}$, where $\mathrm{Q}$ it was called free flow. The filtration process involved the recording of the measured flow values at determined intervals of time (conditioned by the discharge of fluid volume $V=1$ liter) through the filter constituted by the sample fixed in the support of the installation.

Thus, results of the times in which this volume of fluid was discharged were recorded. After $\mathrm{t}=5$ minutes and $23 \mathrm{sec}$ the flow rate $\mathrm{Q}_{\mathrm{i}}=0,1858 \mathrm{l} / \mathrm{min}$ was recorded, where $\mathrm{Q}_{\mathrm{i}}$ is the flow at the beginning of the filtration through the composite sample, and after another 24 minutes a flow rate of $\mathrm{Q}_{\mathrm{s}}=0,041671 / \mathrm{min}$ was recorded, where $\mathrm{Q}_{\mathrm{s}}$ is the flow at the end of 
the filter. In the filtration process there was a variation of the discharged flow. Graphically, this variation was shown in figure 4.

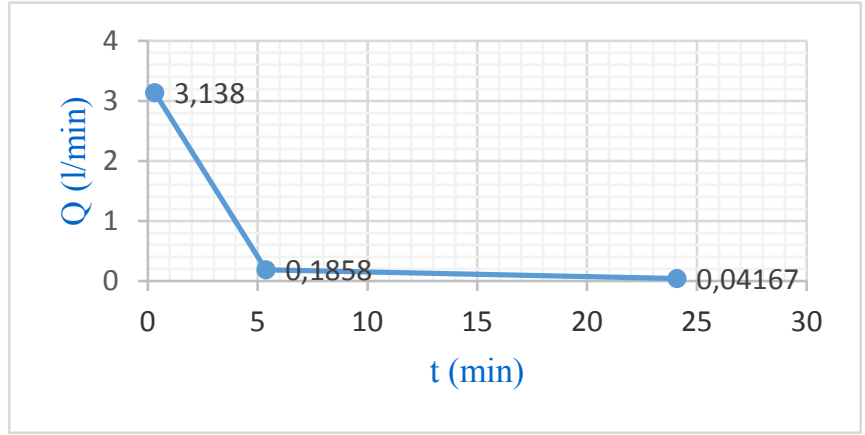

Fig. 4. Flow variation at experiment filtration.

After filtration by composite sample, it was dried under the same initial conditions in order to remove the degree of humidity, then it was weighed analytical and the value of $\mathrm{m}=$ 7,05827 $\mathrm{g}$ was recorded (figure 5).

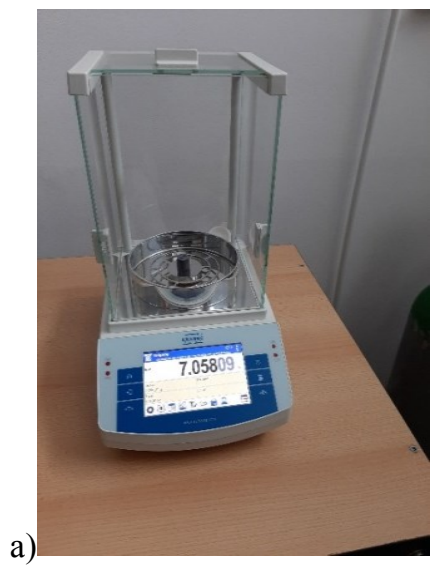

b)

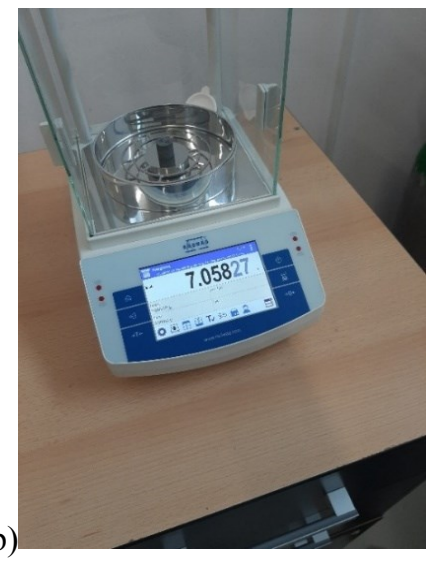

Fig. 5. Analytical weighing of the sample before filtration a) and after filtration b).

The obtain results indicate a mass difference determined by the retentions produced by the composite filter during the filtration process.

\section{Conclusions}

A method and a laboratory installation have been established to accurately measure the filtering capacity of composite materials with ceramic matrix. Determinations were made and results were obtained for certain bentonite-based composites. In this research, a method was undertaken that ensures the possibility of filtering under a pressure gradient ceramic filters obtained based on recipes that offer the possibility of testing them. Filtration through a series of composite materials with very fine pores can be highlighted by using the installation that offers the possibility of ensuring a flow generated by the pressure difference. Following the filtration process, the samples had the ability to retain the solid component of the fluid. It was observed that high flow values were recorded at the beginning of the filtration process. 
During the filtration process, the movement of fluid was due the pressure gradient exerted by the pump of the installation. The fluid volume was directly proportional to the pressure gradient and inversely proportional to the resistance given by the composite filter. The differentiated values of masses of the weighed samples indicated the presence of the solid component retained.

- $\quad$ Sample mass before filtration $\mathrm{m}=7,05809 \mathrm{~g}$

- Sample weight after filtration $\mathrm{m}=7,05827 \mathrm{~g}$

The filter capacity of sample represents a degree of retention of the component solid when a fluid is conveyed through it.

\section{References}

1. C. Kung, C. Hou, Y. Wang, L. Fu, Sens.\&Act. 301, (2019)

2. M. Weiler, US 9938472, B2, (2019)

3. E. Nazarova, D. Alimova, V. Mikhaylov, E. Krisvoshapkina, P. Krivoshapkin, Cer. Int. 45, (2019)

4. S. Kolacek, M. Cerny, Acta Univ. Agr. Silv. Madelianae Brunensis, 61, (2013)

5. S. Zandaryaa, UNESCO Division of Water Sciences. Word Water Week, Global challenge of waste water, (Stockholm, Suedia, 2011)

6. O. San, R. Goren, C. Ogzur, Int. J. Miner. Process, 93, (2009)

7. M.M. Hasan, T. Saeed, J. Nakajima, Envir. Technol.\& Innov., 14, (2019)

8. C. Özgür, O. Șan, Cer. Int. 34, 1935-1939, (2008)

9. J.C. Hyun, J. Kim, H.L. Myong, Cer.Int. 41, (2015)

10. J. Du, H. Zhang, Y. Geng, W. Ming, W. He, J. Ma, Y. Cao, X. Li, K. Liu, Cer. Int., 45, 18155 (2019)

11. K.Man, Q. Zhu, C.Liu, Z. Xing, Cer. Int, 43, (2017)

12. X. Song, B.Jian, J.Jin, Cer.Int., 44, (2018)

13. S. Matei, B. Varga, T. Bedo, M.A. Pop, M. Stoicănescu, A. Crişan, Mat. Today, 19, 1041 (2019)

14. D. Haleem, H. Laan, A. Soppe, S. Heijman, Water R., 124, (2017)

15. C. Salvinelli, A. C. Elmore, M. R. Reidmeyer, K. D. Drake, K. I. Ahmad, Water Res., 104, 28 (2016),

16. M. M. Hasan, M. Shafiquzzaman, M. S. Azam, J. Nakajima, Desal., 276, 272 ( 2011)

17. N. Chaukura, R. Chiworeso, W. Gwenzi, M. Motsa, W. Munzeiwa, W. Moyo, I. Chikurunhe, T.I. Nkambule, Appl. Clay Sci., 185, 105409, (2020)

18. R. Montenegro-Ayo, A.C. Barrios, I. Mondal, K. Bhagat, J. C. Morales-Gomero, M. Abbaszadegan, P. Westerhoff, F. Perreault, S. Garcia-Segura, Scie. of the total Envirom., 737, (2020)

19. G. Negru, Pumping installations and pumps, (ED. Estfalia, Bucharest, 2006)

20. A. Askalany, S. Ernst, P. Hugenell, A. Asaman, Energy, 141, (2017) 